\title{
Adaptive dynamic range matching for spectroscopic measurements
}

\author{
J. Kinast and M. E. Gehm* \\ Electrical and Computer Engineering Department, University of Arizona, Tucson, Arizona 85721, USA \\ ${ }^{*}$ Corresponding author: gehm@ece.arizona.edu
}

Received 17 November 2008; revised 2 March 2009; accepted 6 March 2009; posted 9 March 2009 (Doc. ID 104116); published 25 March 2009

\begin{abstract}
We describe the design and development of an adaptive spectrometer that actively manages dynamic range mismatch between source signals and the spectrometer's detector. The introduction of an inexpensive digital micromirror array yields performance improvements compared to a traditional device. Simulation results are presented that indicate real-world scenarios in which the adaptive spectrometer will yield superior performance. Experimental results from a first-generation prototype adaptive spectrometer confirm the expectations of the simulations. (C) 2009 Optical Society of America
\end{abstract}

OCIS codes: $120.6200,300.6190$.

\section{Introduction}

In many areas of optical detection, one is likely to encounter dynamic range (DR) mismatch, a condition in which the range of signal intensities is ill suited to the range of intensities that can be detected by the sensing device. In some cases, the signal intensities are too weak to use much of the available DR of the detector, while in others the intensities are so great that they drive the detector to saturation. In the most diabolical scenario, both conditions exist at different spatial locations on the detector.

The increasing ubiquitousness of digital sensors has spawned interest in techniques for minimizing the impact of DR mismatch between signals and digital detectors. Given the abundance of digital cameras and the growth of digital video applications in the commercial and industrial sectors, it is not surprising that the majority of DR management efforts have focused on image and video acquisition. For instance, high DR photography is a rapidly growing niche of the community of digital photographers. Here a sequence of digital images of varying exposure times is acquired, and the separate images are combined algorithmically to create a single scene

0003-6935/09/101891-07\$15.00/0

(C) 2009 Optical Society of America in which the number of underexposed and overexposed pixels is minimized [1-8]. DR management techniques have also been applied to video acquisition. In several examples, micromirror arrays have been used to mitigate the impact of saturation in bright regions of the scene of interest [9-11].

DR mismatch does not arise only in image and video acquisition, however. Spectroscopic measurements are also subject to the same issues. The presence of strong spectral features can make it difficult or functionally impossible to detect the presence of weaker spectral features. In contrast to the amount of effort taken to combat DR mismatch for image and video acquisition, there has been surprisingly little effort taken to deal with the problems in the area of spectroscopy. In the simplest approach, multiple exposures are acquired, and strong spectral channels are permitted to saturate. Unfortunately, this "solution" leads to the contamination of spectral channels adjacent to the saturated ones. Another approach is to use a more sophisticated detector that combats blooming effects. While antiblooming detector solutions are becoming increasingly effective [12-17], highly customized detector hardware does not always offer a cost-effective solution to the problem. We present an alternative solution to issues arising from DR mismatch for spectroscopic measurements. We describe the design and performance 
of a prototype adaptive DR spectrometer (ADRS) that uses a digital micromirror (DMM) array to actively match the DR of the spectral source to the DR of the digital detector used in the spectrometer. The DMM array serves as a filter that can turn off the photon flux to spectral channels that would otherwise saturate the detector. The choice of a DMM array as the adaptive component of the ADRS is largely motivated by the increasing use of these devices in consumer electronics (e.g., high-definition televisions and projectors). The resulting availability and decreasing cost of DMM arrays make them an attractive choice for this application.

While DR management techniques in spectroscopy can build on the work that has been performed with images and video, there is at least one critical distinction to be made. In general, when dealing with imagery, we are most concerned with acquiring the canonical "pretty picture." Information about how faithfully the DR of the original scene has been captured and rendered is secondary (at best) to the requirement that the end result be visually pleasing. In contrast, spectroscopic measurements are almost exclusively acquired for quantitative purposes (e.g. analyte detection and concentration estimation) Consequently, when developing DR management strategies for spectroscopic applications, we place more rigorous quantitative requirements on the employed strategies; no information provided by the source signal is discarded in the measurement or analysis stages.

In Section 2 we present a mathematical framework describing the operation and performance parameters of the ADRS. In Section 3 we describe the results of simulations comparing the performance of the ADRS to a traditional spectrometer (TS) that does not employ any DR management strategies. In Section 4 we describe the design and construction of the prototype ADRS before presenting experimental results in Section 5 . Finally, in Section 6 we consider a broader class of designs that rely on the introduction of adaptive components to the spectrometer.

\section{Mathematical Model}

Here we develop a simple mathematical model of the ADRS. A complete measurement sequence involves the acquisition of one or more individual measurements whose exposure times are permitted to vary from measurement to measurement. Once all measurements are acquired, the best estimate of the signal spectrum is generated by using a weighted average of the individual measurements. In addition to suggesting a preferred signal reconstruction method to mitigate the impact of detector readout noise, the model discussed here also forms the mathematical basis of the simulations considered in Section 3 .

Suppose the measurement sequence consists of $k$ measurements. For a multichannel detector, we can represent the $k$ th measurement from the $i$ th channel by

$$
m_{i}^{(k)}=t^{(k)}\left(f_{i}^{(k)} \dot{s}_{i}^{(k)}+\dot{d}\right)+r_{i}^{(k)} .
$$

In Eq. (1), superscripts $(k)$ denote the measurement number, and subscripts $i$ represent the detector channel number. The exposure time is $t$, the photon arrival rate from the spectral source is $\dot{s}$, the dark noise rate from the detector is $d$, and the detector readout noise is given by $r$. The critical feature of the ADRS is that it includes a reconfigurable filter (in our case, a DMM array) that can attenuate the photon flux from the spectral source on a channelby-channel basis. The action of this filter is represented by $f$. For simplicity, we assume that there is a one-to-one correspondence between filter channels and detector channels. Moreover, we make the simplifying assumption that the operation of the filter is perfect and introduces no noise to the measurement process. While it is not necessary that the filter be binary in nature, throughout this paper we assume that $f$ is restricted to the set $\{0,1\}$. The model includes the impact of spectral shot noise (included in $\dot{s})$ as well as the aforementioned detector noise. The detector dark noise $\dot{d}$ is modeled as Poisson noise, and the readout noise $r$ is assumed to be zero-mean additive white Gaussian noise. Note that $\dot{d}$ is assumed to be constant in time and uniform across all detector channels.

We can easily rearrange Eq. (1) to obtain an estimate of the spectral source photon flux from the $k$ th measurement:

$$
\dot{s}_{i}^{(k)}=\frac{1}{f_{i}^{(k)}}\left[\left(\frac{m_{i}^{(k)}-r_{i}^{(k)}}{t^{k}}\right)-\dot{d}\right] .
$$

To obtain a final estimate of the photon flux for a given channel, we construct a weighted average of the $\dot{s}_{i}^{(k)}$ :

$$
\hat{s}_{i}=\frac{\sum_{k} \alpha_{i}^{(k)} \dot{s}_{i}^{(k)}}{\sum_{k} \alpha_{i}^{(k)}} .
$$

If we select $\alpha_{i}^{(k)}=f_{i}^{(k)} t^{(k)}$, then Eq. (3) becomes

$$
\hat{s}_{i}=\frac{\sum_{k}\left(m_{i}^{(k)}-\dot{d} t^{(k)}\right)-\sum_{k} r_{i}^{(k)}}{\sum_{k} f_{i}^{(k)} t^{(k)}} .
$$

The selection of $\alpha_{i}^{(k)}=f_{i}^{(k)} t^{(k)}$ becomes clear if we look at the second sum in the numerator of Eq. (4). If detector readout noise is a zero-mean process (as it is for the present model), then, for a sequence of measurements, the readout noise contributions should sum to zero, on average. This leaves us with a simple expression for estimating the spectral source photon flux for the $i$ th detector channel:

$$
\hat{s}_{i}=\frac{\sum_{k}\left(m_{i}^{(k)}-\dot{d} t^{(k)}\right)}{\sum_{k} f_{i}^{(k)} t^{(k)}} .
$$


Equation (5) offers insight into why the ADRS is effective at reducing the impact of noise on the measurement process. Dark noise can be readily subtracted with simple knowledge of the dark noise rate and the total exposure time. Readout noise (assumed to be zero-mean additive noise) can be mitigated by combining the results of multiple measurements. Most importantly, the presence of the filter allows one to suppress strong spectral features and acquire lengthy exposures while avoiding detector saturation. In the process, the integrated signal and detector dark noise can grow large in comparison to the detector readout noise (which is independent of time). The dark noise is then subtracted computationally, leaving the readout noise and the source signal. By design, the readout noise is then (hopefully) small in comparison to the spectral features that have benefited from the long signal integration time. In sum, the computational subtraction of dark noise and the reduction of the impact of readout noise lowers the overall noise baseline and facilitates the detection of weak spectral features.

\section{Simulations}

To estimate the performance gains one might expect from using an adaptive device, a series of simulations were performed. The simulations were based on the mathematical model discussed in Section 2, and therefore included the impact of spectral source shot noise and detector dark and readout noise. To simplify extraction of relevant information, the spectral source was modeled as having two well-separated peaks whose relative strength was a variable of interest. The photoelectron generation rate due to the weak peak was set to be equal to the dark noise rate of the poorest-performing detector $\left(10 e^{-} / \mathrm{s}\right)$, and the photoelectron generation rate due to the strong peak could be readily varied. For our purposes, we define the DR of the spectral source to be the ratio of the flux of the strong peak to the flux of the weak peak. Further, we define the signal-to-noise ratio (SNR) to be the height of a spectral feature relative to the noise baseline divided by the standard deviation of the noise baseline. We selected a SNR of 5 as the nominal detection/nondetection boundary for the weak spectral feature. For SNR $<5$, positive identification of the weak spectral feature became difficult. While the source spectrum utilized in these simulations is highly artificial and some performance parameters (SNR, photoelectron arrival rate due to the weak spectral feature) are arbitrarily selected, we stress that the conclusions drawn from the simulations are applicable to general spectra.
A series of simulations were conducted that were designed to assess the conditions under which a TS and an ADRS would yield a measurement enabling identification of the weak spectral feature (that is, SNR $\geq 5$ ). To make contact with real-world scenarios of interest, various total measurement times were considered, and a variety of source DRs were explored. The total time budget for making the measurement was allotted differently for the TS and the ADRS. For the TS, each exposure has duration $t_{0}$, which is the longest exposure that can be acquired while avoiding detector saturation. If the total time budget will not permit an integral number of exposures of duration $t_{0}$, then the maximum number of $t_{0}$ exposures are acquired, and the final exposure has a duration $<t_{0}$. For the ADRS, the time budget usage is more complicated. Consider a set of candidate exposure times (CETs) given by $\left\{t_{0}, 2 t_{0}, 4 t_{0}, 8 t_{0}\right.$, $\ldots\}$. The first exposure has duration $t_{0}$. Each subsequent exposure has a duration given by the next longest time in the CETs. This process is repeated until the next longest time in the CETs would exceed the remaining time budget. If any measurement time remains, the longest possible exposure time that would not exceed the time budget is selected from the CETs. Once the time budget dwindles below $t_{0}$, the ADRS sets the exposure time to completely exhaust the time budget. (As an example, suppose the total time budget is $10.5 \mathrm{~s}$ and $t_{0}=1 \mathrm{~s}$. Then the CET set is $\{1,2,4,8, \ldots\}$, and the list of exposure times used by the ADRS will be $1,2,4,2,1$, and $0.5 \mathrm{~s}$.)

To prevent detector saturation in the ADRS, the filter needs to be adjusted after every measurement. Prior to the first measurement, all filter channels are set to transmit all light, that is, $f^{(1)}=1$. For all subsequent measurements, if the anticipated value of $m_{i}^{(k)}$ will exceed the detector's saturation threshold for the chosen $t^{(k)}$, then $f_{i}^{(k)}$ is set to zero for that measurement. As additional exposures are acquired, the estimated photon flux for each spectral channel can be determined more precisely, thereby enabling more intelligent filtering as the measurement sequence progresses.

One of the primary goals of the simulations presented here is to examine how device performance varies with the quality and cost of the detector used in the spectrometer. To this end, simulations were considered for a variety of detectors ranging from a high-end scientific camera to an inexpensive webcam. These detectors and their specifications are listed in Table 1.

The primary results of the simulations are presented in Fig. 1. The four solid and dashed curves

Table 1. Representative Detectors Used in the Simulation

\begin{tabular}{llcrrr}
\hline Detector Class & \multicolumn{1}{c}{ Model } & Bit Depth & Dark Rate $\left(e^{-} / \mathrm{s}\right)$ & Read Noise $\left(e^{-}\right.$RMS $)$ & Cost \\
\hline Scientific & Andor DU434 & 16 & 0.001 & 7.5 & 15 \\
Astrophotography & SBIG ST-7XME & 16 & 1 & 10 & $\$ 25,000$ \\
Midlevel & Lumenera Lu070 & 12 & 10 & 10 & $\$ 2500$ \\
Webcam & - & 8 & 10 & $\$ 200$ \\
\hline
\end{tabular}




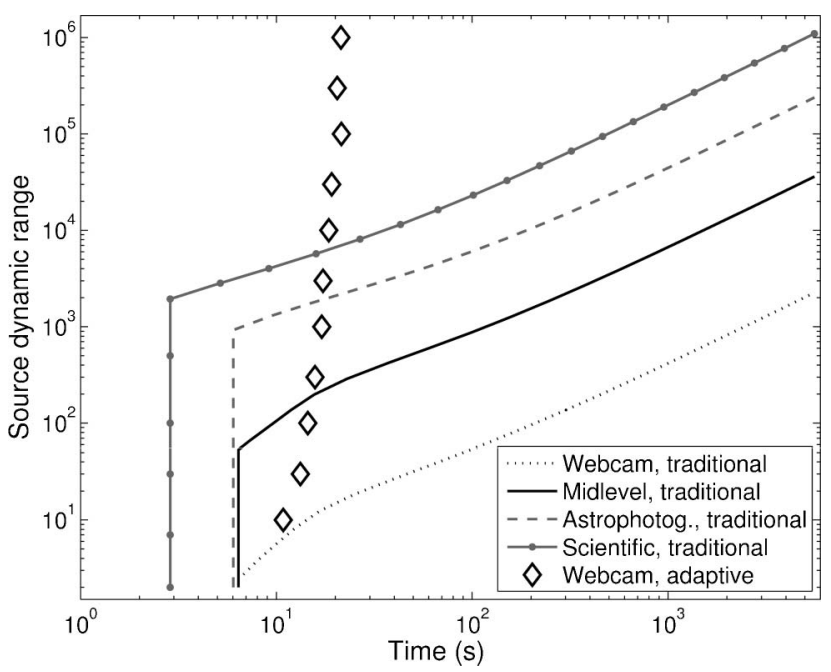

Fig. 1. Simulation results comparing the performance of a TS for four different detectors (solid and dashed curves) to the performance of an ADRS using an inexpensive webcam (open diamonds). Curves represent the nominal detection/nondetection boundary for weak spectral features, with areas below and to the right of the curves indicating measurement parameters permitting successful detection. The ADRS, in spite of using an inexpensive detector, outperforms a TS using more expensive detectors across a broad range of conditions.

represent the detection/nondetection boundary for the weak spectral feature for a TS utilizing the four different detectors mentioned earlier. Areas below and to the right of the curves denote conditions under which the device can successfully detect the weak spectral feature. As one might expect, performance improves when the allotted measurement time is large or when the source DR is sufficiently small. Moreover, the higher grade detectors generally outperform their less expensive counterparts. The diamond points in Fig. 1 denote the nominal detection/nondetection boundary for an ADRS using a generic webcam as a detector. Each of the four curves associated with the TS exhibit a characteristic structure. Each curve begins on the left-hand side of the graph with a vertical line. In this regime, the full $\mathrm{DR}$ of the detector is not in use. Some minimum amount of time (marked by the location of the vertical line) is required for the weak spectral peak to grow above the noise baseline. For each detector being considered, there exists a range of source DRs that can be accommodated by the detector for that particular exposure time. Once the source DR becomes too great, however, the weak spectral peak cannot grow large enough in a single measurement to meet our detection threshold. Multiple measurements must then be acquired and averaged to successfully detect the weak spectral feature. The kink in each curve marks the location at which the simulation switches from a single measurement to a sequence of multiple measurements. Once the simulation enters the multiple measurement regime, the necessary total measurement time increases monotonically with increasing source DR. While all the TS curves exhibit similar structure, the specifications associated with each individual detector accounts for the separation between the curves. The position of the vertical line is impacted solely by the dark noise and readout noise of the detector. Detectors with better noise characteristics can detect the weak spectral feature in a shorter amount of time than their noisier counterparts. The location of the kink is influenced by the detector's noise parameters as well as the bit depth of the detector. As expected, cameras with larger bit depths can accommodate larger source DRs before multiple measurements must be acquired and averaged to meet the detection criteria for the weak spectral feature.

While all the curves associated with the TS assume a similar structure, the smooth pattern associated with the ADRS using the least expensive detector (the diamonds in Fig. 1) is noticeably different. Once the allotted measurement time exceeds approximately $10 \mathrm{~s}$, the ADRS is capable of detecting the weak spectral feature even when the source DR is very large. (Note, however, that in practice the detection of weak features will be improved only if the separation of weak and strong spectral features exceeds the resolution of the adaptive filter in the ADRS.) This should not be surprising, as the larger source DRs are associated with strong photon fluxes that would lead to short initial exposure times to prevent detector saturation. As the source DR increases, the measurement of the strong spectral feature consumes an increasingly smaller portion of the total measurement time budget. (Of course, in reality the ADRS would be incapable of handling source signals of arbitrarily large DR, as hardware limitations would eventually preclude detector exposure times that are sufficiently short to prevent saturation. In addition, short exposure times would lead to problems when attempting to measure spectral sources that vary rapidly in time. However, these same issues also arise for a traditional device utilizing the same detector.) Once the allotted measurement time reaches approximately $18 \mathrm{~s}$, the ADRS with the inexpensive webcam achieves comparable performance to a TS that uses a detector that is roughly 100 times more expensive.

The simulations indicate that the introduction of an adaptive component to a spectrometer can substantially improve performance across a wide range of measurement conditions. To verify this conclusion, a prototype ADRS was designed, constructed, and tested. The development and testing of the prototype is discussed in Sections $\underline{4}$ and $\underline{5}$.

\section{Device Design}

A simplified schematic of the optical design of the ADRS is provided in Fig. 2. For simplicity, the design can be considered in two parts. The first part of the design is a normal dispersive spectrometer utilizing a reflective diffraction grating. However, where one would normally place a detector array, we have substituted a DMM array. The second part of the 


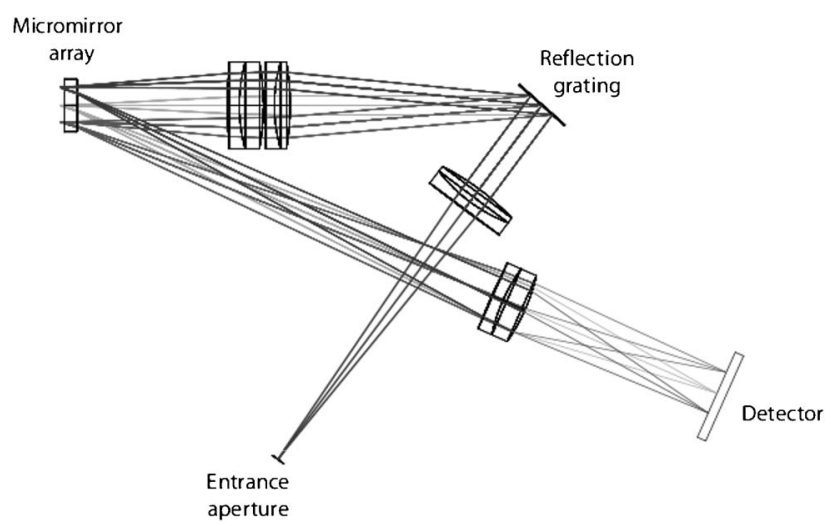

Fig. 2. Schematic of the optical design of the ADRS. Light from an input slit is collimated onto a reflective diffraction grating. A DMM array is located at the focal plane of input slit. The surface of the DMM array is then reimaged onto a detector. In the schematic, all spectral channels are in the "on" position, in which photons are directed to the detector rather than a beam dump (not shown).

optical design images the surface of the DMM array onto a detector array. The remainder of this section offers further details about design choices and considerations.

Nominally, the first half of the optical design is a simple dispersive spectrometer with a $4 f$ lens configuration. (Optimization to reduce spot sizes at the DMM array results in departures from a true $4 f$ configuration.) Light from a $10 \mu \mathrm{m}$ input slit is collimated onto a reflective diffraction grating designed for use with visible wavelengths. Light from the first diffraction order passes through a second set of lenses and is imaged onto the DMM array. In a traditional dispersive spectrometer, the detector would be placed at the DMM array location and the desired spectrum would be measured. However, for the ADRS, photons striking the DMM array are reimaged onto a detector array after further propagation. The location of the final detector array is based on several considerations. First, the detector position is chosen such that the image of the DMM array is in focus on the detector. Second, the micromirrors that comprise the DMM array have three discrete orientations. When the DMM array is not powered, the surface of each mirror is parallel to the surface of the underlying substrate. That is, the DMM array acts like a "normal" mirror. When the DMM array is powered, there are two possible mirror orientations, corresponding to rotations of $\pm 12^{\circ}$ away from the relaxed position. As a result of these orientation limitations, the position of the final detector array is chosen such that the "on" mirrors will direct their photons to the detector array while the "off" mirrors will send their photons to a beam block (not shown in Fig. 2). The optical design of the ADRS proceeded in two stages. First, design parameters for all optics prior to the DMM array were optimized to minimize spot sizes at the DMM array. Second, while holding all the pre-DMM array design parameters constant, parameters for all optics located after the DMM array were optimized to minimize spot sizes at the detector plane. Handling the design in this fashion ensures best utilization of the spatial resolution of both the DMM array and the detector. Given the presence of multiple reflective optical components in the design, along with the multitude of configurations possible with the DMM array, care was taken to limit the propagation of stray light during the optical design stages. ZEMAX was used for all optical design tasks.

Design of optomechanical mounts was performed in SolidWorks, and the resulting structures were fabricated on an Eden 350 rapid prototyping machine. Rather than creating a monolithic structure, independent mounts were created and fastened to an optical table. While several lens positions were adjustable, successful alignment of the device generally relied on altering the positions of the DMM array and detector using translation stages. All lenses and other optics were off-the-shelf components. The detector was a Unibrain Fire-i XGAb camera, and the DMM array was extracted from a budget ViewSonic PJ503D projector. To achieve control of the DMM array, we utilized the projector's video graphics array (VGA) interface along with a MATLAB script that enabled display of static images on a secondary display. Additionally, MATLAB was used to control the detector, calculate the optimal DMM array configuration during the measurement, and conduct all analysis of the resulting data.

Successful operation of the ADRS requires knowledge of how the mirrors on the DMM array map to the pixels on the detector. This was accomplished by placing a broadband light source at the spectrometer's input slit and using the VGA interface to display a simple test pattern of five numbered dots on the DMM array (one dot at each corner of the active mirror region and one in the center of the active region). This test pattern was then recorded by the detector, and the locations of the numbered dots were identified on the recorded image. Using the correspondence between these five locations, built-in MATLAB routines for affine transforms and interpolation were employed to map individual DMM array mirrors to detector pixels. Finally, due to differing mirror and pixel sizes as well as optical design constraints, there is not a one-to-one mapping between DMM array mirrors and detector pixels. In this particular design, the DMM array mirrors are the limiting factor in the device's spectral resolution.

\section{Experimental Results}

To test the performance of the prototype ADRS, a Pen-Ray neon gas lamp was utilized as a spectral source. A total time budget of $5 \mathrm{~s}$ was considered, and the usage of that time budget as well as the updating of the DMM array filter configuration was conducted according to the specifications outlined in Section 3. Signal reconstruction was performed using Eq. ( $(\overline{5})$. In addition to operating the ADRS in the adaptive mode, a "traditional" mode could be achieved by setting all the mirrors on the DMM 
array to transmit all light. (When operating in traditional mode, however, all exposure times were fixed at $t_{0}$, the maximum exposure duration that did not result in detector saturation.)

In Fig. 3 we show detector images from the prototype ADRS for two exposure times. The upper panel shows the initial exposure $(t=0.07 \mathrm{~s})$, while the lower panel corresponds to an exposure time of $1.12 \mathrm{~s}$. One can easily see that the strong spectral features that appear in the short exposure have been suppressed during the longer exposure, enabling the detection of weaker features at other wavelengths. Note also that there are vertical discontinuities in the spectral lines in the longer exposure. These gaps correspond to locations where the ADRS has determined that the estimated photoelectron count for that exposure would locally exceed the detector's well depth. This condition contrasts with expectations for an ideal system, in which one would expect the ADRS to turn off the entire spectral line for a given exposure. However, imperfections such as dust particles at the spectrometer's input slit, "hot" pixels on the detector, statistical fluctuations due to shot noise, and varying optical transmission efficiency as a function of vertical position on the slit create a more complex situation. As a result, the on/off status of each micromirror on the DMM array is determined on a mirror-by-mirror basis.

Figure 4 compares the performance of the ADRS to the performance of the TS using the same detector and a measurement time budget of $5 \mathrm{~s}$. There were eight exposures for the ADRS with exposure times ranging up to $2.24 \mathrm{~s}$. For the TS, 71 exposures of duration $0.07 \mathrm{~s}$ were acquired, along with one exposure of duration $0.03 \mathrm{~s}$, and averaged. For both the TS
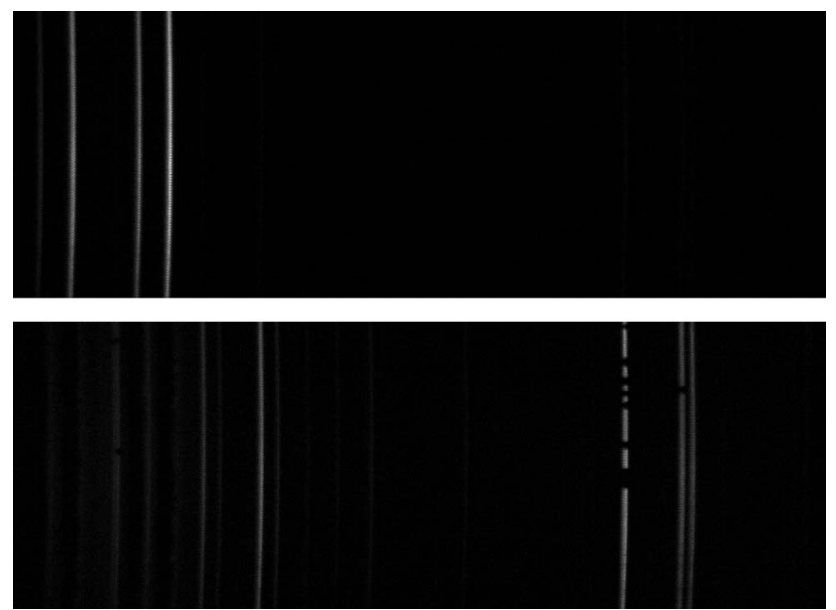

Fig. 3. Detector images demonstrating the spectrometer's ability to suppress strong spectral features as the signal acquisition time is varied. The exposure time for the upper panel is $0.07 \mathrm{~s}$. The exposure time for the lower panel is $1.12 \mathrm{~s}$. Note that the strong spectral features on the upper panel have been suppressed in the lower panel, enabling a longer exposure time and improved SNR for weaker spectral features. The vertical discontinuities in the spectral lines in the lower panel arise because the ADRS has estimated that the photoelectron count for that exposure would locally exceed the detector's well depth.

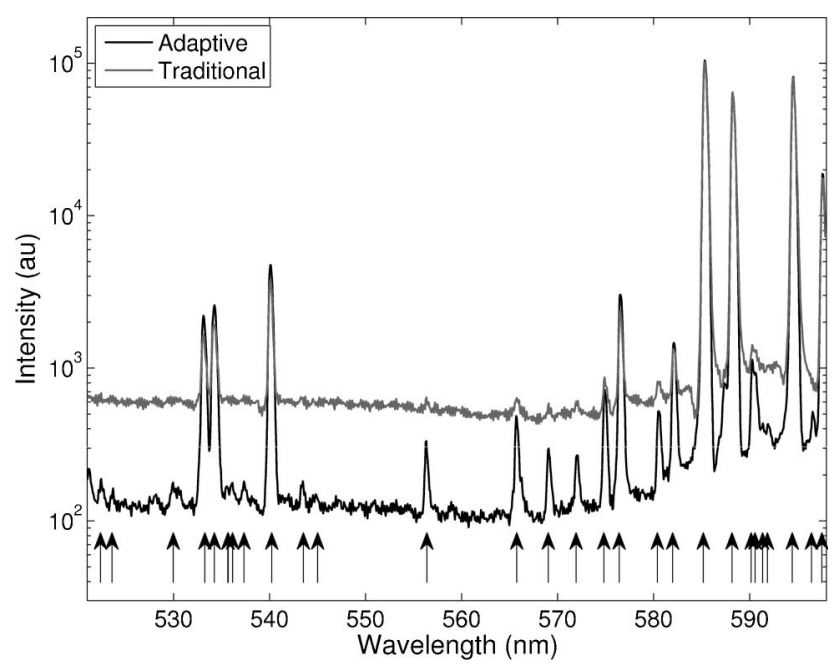

Fig. 4. Measured neon spectra when operating the spectrometer in the adaptive mode (black) and traditional mode (gray) using the same total time budget. In both modes, detection of strong spectral features is good. However, the adaptive spectrometer exhibits a smaller noise baseline, enabling improved detection of weak spectral features. Arrows indicate the positions of known lines in the neon spectrum.

and the ADRS, detection of strong spectral features is good. However, the ADRS exhibits a lower noise baseline. Recall from the discussion at the end of Section 2 that the lower noise baseline is possible because the ADRS uses a weighted average of long exposures to computationally reduce the impact of detector dark and readout noise. This enables improved detection of weak spectral features throughout the spectral range of the ADRS. Arrows in the figure denote the positions of known lines in the neon spectrum. Because of differing detector noise specifications, we omit a direct comparison of our experimental results to the simulations presented in Section 3 . However, the prototype is intended as a proof of concept, and we can obtain an estimate of the improvement in DR by considering the drop in the noise baseline when switching from traditional to adaptive measurement modes. We estimate the DR by calculating the ratio of flux due to the strongest spectral feature (relative to the noise baseline) to the flux due to a hypothetical spectral feature whose peak rises five standard deviations above the noise baseline. (Recall that SNR $=5$ is our nominal detection/nondetection boundary for a weak spectral feature.) When operating the spectrometer in traditional mode, we estimate a DR of roughly 142. When operating the spectrometer in the adaptive mode, we estimate a DR of roughly 651 , more than 4.5 times the DR for the traditional mode. These results confirm the findings of the simulations: the introduction of an adaptive component to the spectrometer design facilitates the measurement of spectral sources with larger DRs.

\section{Conclusion}

We have described a novel spectrometer design that incorporates an adaptive filter component. The 
adaptive filter enables active DR matching between a spectral source and the spectrometer's detector. Its inclusion allows a spectrometer using an inexpensive digital detector to achieve performance parity with a traditional device that utilizes a far more expensive detector. Simulations indicate that these performance improvements can be realized for a wide range of measurement conditions. The development and testing of a first-generation ADRS prototype confirms that the adaptive filter enables DR management for spectroscopic measurements.

We utilized a limited subset of the DMM array's capabilities. We implemented only a binary filter, though the DMM array is readily capable of performing grayscale filtering as well. Moreover, the general spectrometer architecture developed here can be modified to facilitate more exotic, multiplexed measurement strategies. These directions are currently under exploration in our laboratory.

\section{References}

1. P. Burt and R. J. Kolczynski, "Enhanced image capture through fusion," in Proceedings of the 4th International Conference on Computer Vision (IEEE, 1993), pp. 173-182.

2. B. C. Madden, "Extended intensity range imaging," Tech. Rep. (GRASP Laboratory, University of Pennsylvania, 1993), pp. $1-19$.

3. C. Schlick, "Quantization techniques for visualization of high dynamic range pictures," in 5th Eurographics Workshop on Rendering (Springer-Verlag, 1994), pp. 7-18.

4. S. Mann and R. W. Picard, "Being 'undigital' with digital cameras: extending dynamic range by combining differently exposed pictures," in Proceedings of the 46th Annual IS\&T Conference (IEEE, 1995), pp. 442-448.

5. P. E. Debevec and J. Malik, "Recovering high dynamic range radiance maps from photographs," in Proceedings of the 24th Annual Conference on Computer Graphics and Interactive Techniques (ACM Press/Addison-Wesley, 1997), pp. 369-378.
6. T. Mitsunaga and S. K. Nayar, "Radiometric self calibration," in IEEE Computer Society Conference on Computer Vision and Pattern Recognition (IEEE, 1999), Vol. 1, pp. 374-380.

7. M. A. Robertson, S. Borman, and R. L. Stevenson, "Dynamic range improvement through multiple exposures," in Proceedings of the International Conference on Image Processing (IEEE, 1999), Vol. 3, pp. 159-163.

8. S. Battiato, A. Castorina, and M. Mancuso, "High dynamic range imaging for digital still camera: an overview," J. Electron. Imaging 12, 459-469 (2003).

9. J. Castracane and M. Gutin, "DMD-based bloom control for intensified imaging systems," Proc. SPIE 3633, 234-242 (1999).

10. M. P. Christensen, G. W. Euliss, M. J. McFadden, K. M. Coyle, P. Milojkovic, M. W. Haney, J. van der Gracht, and R. A. Athale, "ACTIVE-EYES: an adaptive pixel-by-pixel imagesegmentation sensor architecture for high-dynamic-range hyperspectral imaging," Appl. Opt. 41, 6093-6103 (2002).

11. S. K. Nayar and V. Branzoi, "Adaptive dynamic range imaging: optical control of pixel exposures over space and time," in Proceedings of the 9th International Conference on Computer Vision (IEEE, 2003), Vol. 2, pp. 1168-1175.

12. S. C. Wang and D. N. Ludington, "Readout circuit for optical sensing charge injection device facilitating an extended dynamic range," U.S. patent 4,734,776 (29 March 1988).

13. S. Mendis, S. Kemeny, R. Gee, B. Pain, Q. Kim, and E. Fossum, "Progress in CMOS active pixel image sensors," Proc. SPIE 2172, 19-29 (1994).

14. Y. T. Tsai, "Method and apparatus for extending the dynamic range of an electronic imaging system," U.S. patent 5,309,243 (3 May 1994).

15. E. P. Rudd and T. A. Skunes, "Method and apparatus for exposure control in light-based measurement," U.S. patent 5,519,204 (21 May 1996).

16. V. M. Brajovic, R. Miyagawa, and T. Kanade, "Temporal photoreception for adaptive dynamic range image sensing and encoding," Neural Networks 11, 1149-1158 (1998).

17. M. A. Tan and J. Luo, "Self-calibrating anti-blooming circuit for CMOS image sensor having a spillover protection performance in response to a spillover condition," U.S. patent 7,381,936 (3 June 2008). 